\title{
Philosophiques
}

\section{Emmanuel Renault, Marx et la philosophie, Paris, PUF, coll. "Actuel Marx Confrontation », 2014, 207 p.}

\section{Arnaud Theurillat-Cloutier}

Volume 42, numéro 1, printemps 2015

URI : https://id.erudit.org/iderudit/1032224ar

DOI : https://doi.org/10.7202/1032224ar

Aller au sommaire du numéro

Éditeur(s)

Société de philosophie du Québec

ISSN

0316-2923 (imprimé)

1492-1391 (numérique)

Découvrir la revue

Citer ce compte rendu

Theurillat-Cloutier, A. (2015). Compte rendu de [Emmanuel Renault, Marx et la philosophie, Paris, PUF, coll. "Actuel Marx Confrontation », 2014, 207 p.]

Philosophiques, 42(1), 193-197. https://doi.org/10.7202/1032224ar

Ce document est protégé par la loi sur le droit d'auteur. L'utilisation des services d’Érudit (y compris la reproduction) est assujettie à sa politique d'utilisation que vous pouvez consulter en ligne.

https://apropos.erudit.org/fr/usagers/politique-dutilisation/ 


\title{
Comptes rendus
}

\author{
Emmanuel Renault, Marx et la philosophie, Paris, PUF, coll. «Actuel \\ Marx Confrontation », 2014, 207 p.
}

Dans ce nouvel ouvrage, Emmanuel Renault expose le parcours tortueux de Marx dans son rapport à la philosophie ${ }^{1}$. À l'exception de trois chapitres et de la conclusion, le livre reprend pour l'essentiel des interprétations déjà développées par l'auteur dans des articles publiés entre I999 et 2012. Elles sont ici présentées sous une forme plus développée, et intégrées à un tout cohérent divisé selon trois axes. Dans la première partie, l'auteur interroge les grands concepts qui ont divisé les héritiers de Marx quant au statut de sa philosophie. La deuxième partie se propose d'exposer le moment ouvertement philosophique de la pensée de Marx, soit sa période de jeunesse. En dernier lieu, l'auteur présente le fruit de la critique marxienne de la philosophie, c'est-à-dire sa traduction en une critique de l'économie politique, en tentant d'y relever la permanence des enjeux philosophiques. Rares sont les ouvrages des études marxiennes comme celui-ci, qui allient à la fois rigueur et clarté d'exposition, mise en perspective des débats précédents ayant animé marxistes et marxiens de langues française, allemande et anglaise, et maintien d'une interprétation riche et complexe de la pensée de Marx, au-delà des lectures parfois sélectives et unilatérales qui frisent l'apologie.

Marx était-il philosophe ou théoricien des sciences sociales? Cette question introductive paraît insoluble sans clarifier la conception invoquée de la philosophie, mais plus encore sans préciser de quels moments de la pensée de Marx il est question. Au-delà des lectures «continuiste ${ }^{2}$ » (Engels, Plékhanov, Michel Henry ${ }^{3}$, etc.) ou "discontinuiste»(Althusser), qui, ou bien sous-estiment l'originalité des textes de maturité, ou bien négligent la prégnance des thèses de jeunesse dans les œuvres postérieures, Renault s'attarde à restituer le contexte à partir duquel Marx a développé sa compréhension de la philosophie pour mieux livrer le sens de son projet de «sortie de la philosophie». Le résultat serait davantage une nouvelle pratique de la philosophie

1. Cet ouvrage paraît en même temps que la réédition de l'utile introduction à l'œuvre de Marx, qui peut allègrement se lire en complément de l'ouvrage recensé ici: Gérard Duménil, Michael Löwy, et Emmanuel Renault, Lire Marx, Paris, PUF, 20 I 4.

2. Emmanuel Renault, Marx et la philosophie, Paris, PUF, coll. «Actuel Marx Confrontation ", 20I4, p. 9.

3. Cette catégorisation paraît peut-être trop englobante, puisque les raisons profondes de ces lectures continuistes divergent profondément. On notera également que la présence de Michel Henry dans cette série semble plutôt surprenante dans la mesure où, relativement à la thèse classique d'Althusser de la "coupure épistémique ", Michel Henry situe également, bien que pour d'autres raisons qu'Althusser, l'avènement de la véritable philosophie de Marx à partir de l'Idéologie allemande, rejetant de ce fait les Manuscrits de I 844 et les textes précédents dans les épaves de l'hégélianisme et du feuerbachisme. 
qu'une nouvelle philosophie elle-même. À la thèse défendue par Étienne Balibar $^{4}$ selon laquelle il y aurait plusieurs philosophies de Marx, il ajoute que ces diverses philosophies peuvent se comprendre à la lumière d'une évolution exploratoire procédant chaque fois par autocritique du moment antérieur. Ultimement, la critique de la philosophie aboutirait, non à un rejet total de celle-ci, mais plutôt à une conception déflationniste de la philosophie: abandonnant une position maximaliste (dont le point culminant serait les Manuscrits de I 844), Marx récuserait l'autonomie de la philosophie pour ne retenir d'elle qu'une série d'instruments fondamentaux (autoréflexion, synthèse, analyse critique) devant être associés aux sciences positives (économie politique, sociologie, histoire, etc.), et à la pratique sociale. Selon Emmanuel Renault, la pratique contemporaine de la philosophie aurait tout à gagner de cette leçon marxienne de relativisation: cela lui permettrait de continuer à valoir comme forme de discours rationnel sans se bercer d'illusions sur sa supériorité relativement aux autres formes de rationalités différenciées; elle ne s'envisagerait plus comme un cadre théorique fixe, mais plutôt comme une démarche d'autoréflexion sur des savoirs et pratiques sociales en transformation permanente; elle renoncerait à prétendre pouvoir connaître le monde social uniquement par ses propres moyens; enfin, elle éviterait les écueils possibles d'une défense d'un devoir-être complètement abstrait des luttes sociales existantes. Notons cependant que la raison pour laquelle il faudrait absolument se tenir dans la dichotomie entre nouvelle pratique de la philosophie et nouvelle philosophie n'apparaît pas suffisamment claire. Cette nouvelle pratique marxienne nous semble être le résultat de thèses proprement philosophiques (par exemple, celle de la non-autonomie de la philosophie), de même que cette pratique continue, par sa mise en œuvre, à perpétuer et à défendre une certaine philosophie, qu'on pourrait appeler, en suivant Franck Fischbach, une philosophie sociale ${ }^{5}$.

Dans le chapitre portant sur le concept de critique, les diverses fonctions de celui-ci sont exposées, condensant en quelques pages un problème déjà traité plus en longueur par l'auteur dans un précédent ouvrage ${ }^{6}$. Dans sa première version, la critique marxienne n'aurait rien d'original quant à son principe, comparée à la critique kantienne de la métaphysique et àala critique feuerbachienne de la religion, puisque sa fonction de démystification de la conscience ne ferait que s'appliquer à des objets différents et selon d'autres intentions. Par la suite, Marx serait passé à une conception pragmatique de la critique, la reléguant au rôle d'instrument au service de la pratique, pratique elle-même occupée à surmonter des problèmes identifiés grâce à l'enquête empirique, et non à partir d'abstractions philosophiques.

4. Etienne Balibar, La philosophie de Marx, Paris, La Découverte, $3^{\mathrm{e}}$ éd., 2010.

5. Franck Fischbach, Manifeste pour une philosophie sociale, Paris, La Découverte, 2009.

6. Emmanuel Renault, Marx et l'idée de critique, Paris, PUF, I995. 
Enfin, dans ses travaux de maturité, la critique serait introduite à même l'économie politique afin de produire un discours qui, tout en prétendant à la vérité, permettrait d'expliquer les erreurs des autres discours.

Le chapitre consacré à la dialectique a essentiellement une visée négative: il tente de disqualifier les diverses tentatives marxistes d'interprétation de la philosophie de Marx à l'aune du concept de dialectique. Après avoir rappelé la distinction entre le dialectique et la dialectique dans la philosophie de Hegel, et écarté certaines thèses faussement attribuées par les marxistes à ce dernier, Renault procède à une critique d'Engels, fidèle compagnon de Marx, qui aurait toutefois eu la maladresse de fixer les termes obtus du marxisme. L'auteur rejette l'idée de dialectique matérialiste, en faisant valoir que son réductionnisme naturaliste, qui implique notamment l'assimilation de la pensée à un simple reflet des processus naturels, semble incompatible avec la position de Marx. Il attribue les dérives théoriques postérieures du marxisme (Kautsky, Bernstein) à la réduction engelsienne de la dialectique à une série de lois du changement et au cantonnement de la philosophie à un rôle de synthèse des sciences de la nature. Évidemment, d'autres penseurs avaient déjà tenté de critiquer Engels afin de redorer le blason de Marx au xx ${ }^{e}$ siècle (Luckàcs, Korsch). Par contre, en faisant de la dialectique hégélienne l'opérateur de ce renouvellement, ils auraient, selon la démonstration de Renault, attribué à Hegel des thèses qui lui étaient étrangères, en plus d'avoir procédé à une sélection arbitraire des différentes références de Marx à la dialectique.

Il rappelle que le rapport de Marx à Hegel est d'abord à comprendre sous l'angle de la critique, qui confère à la plupart des références explicites à la dialectique une connotation péjorative. Si, à partir des Grundrisse (I857-I 858), la dialectique est bel et bien venue jouer un rôle dans la critique de l'économie politique, elle n'a jamais constitué le supposé principe métaphysique de toute chose. Quant au Capital, devant l'impossibilité d'y déceler une conception unifiée des divers usages de la dialectique (polémique, méthodique, révolutionnaire), il faudrait se résoudre à y reconnaître l'absence de méthode prédéterminée au profit d'une enquête scientifique aux opérateurs logiques pluriels, certes parfois empruntés à Hegel, mais sans qu'ils ne forment jamais un système de lois. L'organisation générale de l'ouvrage peut bien être placée sous l'épithète de "dialectique ", mais ce même qualificatif ne peut être attribué à l'organisation des chapitres, ni à la construction et à l'articulation des concepts, qui obéissent à des nécessités logiques hétérogènes.

Dans le dernier chapitre consacré aux modèles critiques, l'auteur se penche sur l'épineuse question du rapport de Marx à la normativité. Il rappelle que la spécificité de la critique marxienne du capitalisme se loge en partie dans le fait qu'elle n'a jamais recours à des normes morales universelles. Cette position s'expliquerait à son avis en vertu de quatre grandes raisons invoquées par Marx: la variation des valeurs en fonction des évolutions 
sociales invite au scepticisme moral; la vacuité et le formalisme des devoirs moraux les rendent souvent indéterminés (critique hégélienne de Kant); d'un point de vue pragmatique, les principaux moraux ont peu d'emprise sur l'histoire, contrairement aux intérêts et aux besoins; enfin, le caractère antagonique de la dynamique historique (la lutte des classes) est inconciliable avec un discours éthico-moral universaliste. Ainsi, en faisant de sa critique de l'économie politique le « représentant ${ }^{7}$ » du prolétariat, Marx aurait cherché à définir sa position théorique non pas de manière abstraite et universaliste, mais en prenant pour appui les luttes effectives de la partie de la société dont l'oppression constituait le noyau. Si, dans ses références au communisme, Marx semble s'appuyer sur une définition de la justice et une éthique du développement intégral de l'individualité, Renault soutient qu'il évite la contradiction en écartant d'une part toute référence au devoir-être dans son analyse de l'action historique, prenant plutôt pour guide l'expression normative immanente du besoin, et, d'autre part, en ne faisant jamais intervenir sa conception de la justice et de l'éthique dans les principes qui animent la critique du monde capitaliste.

La deuxième partie est consacrée à trois moments de la formation philosophique de Marx. Le chapitre consacré au mouvement jeune-hégélien constitue une précieuse mise en perspective du contexte intellectuel du jeune Marx. Renault insiste beaucoup, contre les lectures de Mercier-Josa et d'Althusser, sur la nécessité de lire les textes de jeunesse à l'intérieur des référents de ce mouvement politico-intellectuel, animé entre entres par Bauer, Ruge et Feuerbach. Cette restitution philologique permet de distinguer le jeunehégélianisme à l'intérieur de la gauche hégélienne par sa volonté de répondre à trois grandes questions: "Quelle est la signification historique de la philosophie hégélienne? Comment sa philosophie doit-elle être transformée? Quelle est la meilleure manière de rendre la philosophie hégélienne utile à la rationalisation du monde historique ${ }^{8}$ ? » L'originalité du jeune Marx se trouverait alors dans son appréciation de Hegel comme penseur tant du passé que de l'avenir, dans sa volonté de dépasser les limites du système hégélien en développant ses contradictions internes, et dans l'affirmation de l'impossibilité de réaliser la philosophie sans la nier, et inversement.

Dans la troisième et dernière partie, Emmanuel Renault se penche sur la contribution de Marx à l'économie politique. Contrairement à la lecture de la Wertkritik (Robert Kurz, Anselm Jappe, Moishe Postone), Renault défend que l'œuvre de Marx ne doit pas être lue unilatéralement comme une critique sans reste de l'économie politique, mais plutôt comme une « refonte de l'économie politique classique ${ }^{9}$ » opérée par l'historicisation de ses caté-

7. Karl Marx, Le capital, livre premier, Paris, PUF, I993, trad. Jean-Pierre Lefebvre, p. I3.

8. Ibid., p. 89 .

9. Renault, Marx et la philosophie, p. I49. 
gories et l'intégration d'une analyse sociale. Cette interprétation a le mérite de ne pas tenter d'écarter les affirmations pourtant explicites de Marx sur la question. Le chapitre portant sur le travail et la domination restitue, quant à lui, les perspectives multiples sur le «rapport social de domination». Renault défend que Marx aurait été le premier à concevoir la domination des individus comme une forme de personnification des positions sociales qui contraint les acteurs à reproduire la structure sociale pour survivre. Ainsi, la spécificité du capitalisme devrait se comprendre par la prédominance des contraintes objectives indirectes de l'économie (travailler pour survivre), mais cette caractéristique n'abolirait pas selon Marx les autres formes de contraintes directes et personnalisées (contrairement à ce que défendent parfois les tenants de la Wertkritik).

Dans la même veine, Renault invite à dépasser l'opposition abstraite entre les lectures qui font de Marx un critique $d u$ travail, et celles qui défendent qu'il opérerait une critique par le travail. La critique de la domination à l'intérieur de la sphère du travail semble en fait indissociable de la critique de la domination sociale par le travail abstrait. Autrement dit, sortir du mode de production capitaliste signifierait tout autant réduire le temps de travail qu'émanciper le travail, le premier étant la condition du second, dans la mesure où la démocratisation des lieux de travail suppose de libérer $\mathrm{du}$ temps pour la formation intellectuelle et technique des ouvriers, indispensable pour les rendre maitres de l'organisation de la production.

Le chapitre consacré à la critique du marché constitue, quant à lui, une bonne mise au point à propos de l'objet véritable d'enquête de Marx. Renault rappelle que l'œuvre de Marx ne doit pas être comprise comme une théorie critique du marché proprement dite, car Marx jugeait ce concept bien trop hétérogène. À l'heure où la question de la redistribution et du marché semble centrale dans les débats (Rawls, Honneth), un court détour par Marx peut convaincre que l'essentiel se joue plutôt du côté de la production et de ses contradictions.

Dans ce contexte, quelle place resterait-il pour la philosophie dans la critique de l'économie politique de Marx? Selon Renault, elle persisterait dans les finalités (connaissance et transformation de la société) et les moyens de son activité théorique (réflexion critique sur la métaphysique de l'économie politique, exposition critique de ses catégories, et autoréflexion méthodologique). Cette inflexion de la philosophie vers la théorie sociale est loin de pouvoir rallier tous ceux qui se revendiquent de la philosophie. Malgré cela, l'ouvrage de Renault exprime très bien pourquoi, dans l'histoire de la philosophie, il semble aujourd'hui difficile de faire fi du "moment Marx » pour défendre sa propre orientation philosophique.

ARNAUD THEURILLAT-CLOUTIER professeur de philosophie au collégial 